\title{
Effects of Hematocrit of the Sealed Blood on the Leakage Characteristics of Blood Cell Component and Plasma Component in a Mechanical Seal
}

\author{
Jun Tomioka $^{1)^{*}}$, Mikiko Oyabu ${ }^{2)}$ and Norifumi Miyanaga ${ }^{3)}$ \\ ${ }^{1)}$ Waseda University \\ 3-4-1 Okubo, Shinjuku-ku, Tokyo 169-8555, Japan \\ ${ }^{2)}$ Graduate School of Waseda University \\ 3-4-1 Okubo, Shinjuku-ku, Tokyo 169-8555, Japan \\ ${ }^{3)}$ Kanto Gakuin University \\ 1-50-1 Mutsuura Higashi, Kanagawa-ku, Yokohama, Kanagawa 236-8501, Japan \\ *Corresponding author: tomioka@waseda.jp \\ ( Manuscript received 30 October 2015; accepted 30 March 2016; published 15 September 2016 ) \\ ( Presented at the International Tribology Conference Tokyo 2015, 16-20 September, 2015)
}

\begin{abstract}
The purpose of this study is to find out the effects of hematocrit of the sealed blood on the leakage characteristics of blood cell components and plasma components in a mechanical seal, which separates blood from cooling water. The hematocrit was controlled to 0, 10, 20, 30 and 40\%. Each component leakage was obtained by measuring the increase in the potassium ion concentration and the sodium ion concentration in the cooling water. It was revealed that each component leakage became larger with the increase of the hematocrit in the three-hour measurement. When changing the hematocrit from $20 \%$ to $30 \%$, the volume percentage of the blood cell component leakage remarkably increased by 7.7 times. The friction characteristics of the mechanical seal under blood sealing and the hemolytic properties of the sealed blood were also studied. There is a typical trend under the mixed lubrication condition where the friction torque decreased with each of the sealed blood as the rotational speed increased. The hemolytic index of the sealed blood after the measurement experiments of the blood leakage increased with the increase of the hematocrit.
\end{abstract}

Keywords: mechanical seal, hematocrit, leakage characteristics, friction characteristics, hemolytic properties, blood cell components, plasma components

\section{Introduction}

In left ventricular assist devices with a continuousflow rotary pump, a design of blood seal is very important for the long-term support. The major issues in these parts are to reduce the thrombus formation because of the frictional heat generation and the mechanical wear. Yamazaki, Tomioka et al. [1,2] developed a rotary blood pump with the Cool-Seal system where a mechanical seal controlled blood leakage into the motor side. In this system, the cooling water (C. W.), which cooled, washed and lubricated the sealing faces of the mechanical seal, was circulated to reduce the frictional heat generation. As a result, the mechanical wear of the sealing faces was less than $0.05 \mu \mathrm{m}$ a day so that the durability for the long-term support more than 10 years of the device was revealed [2]. Tomioka et al. [3,4] also examined experimentally that the lubrication characteristics of the mechanical seal with the Cool-Seal system and that the effects of the surface roughness of the sealing faces on the lubrication characteristics of the mechanical seal under blood sealing. They obtained the blood leakage by measuring the increase in the sodium ion concentration in the C. W. because the sodium ion is mostly contained in blood. In this method, however, it was not considered that blood consists of blood cell components and plasma components and that the ion composition in each component is different. It was also assumed that the volume percentage of blood cell components in the blood passing between the sealing faces is equal to the volume percentage of blood cell components in the sealed blood, that is, the hematocrit. However the film thickness in the sealing faces of the mechanical seal is considered to be less than $1 \mu \mathrm{m}$ [5] and it is supposed to be smaller than the blood cell component represented by red blood cells having a width of $8 \mu \mathrm{m}$ and a thickness of $2 \mu \mathrm{m}$. Therefore the mechanism of the blood leakage phenomenon could not be explained with the previous method where the volume percentage of the blood cell component leakage is assumed to be equal to the 
hematocrit of the sealed blood. To find out the blood leakage phenomenon, it is needed to reveal each leakage characteristics of blood cell components and plasma components. Tomioka and Oyabu et al. [6], therefore, proposed a new measurement method of each component leakage from the increase in the sodium ion concentration and the potassium ion concentration in the C.W. and found out that the volume percentage of the blood cell component leakage was not equal to the hematocrit of the sealed blood.

The purpose of this study is to reveal each leakage characteristics of blood cell components and plasma components in detail, conducting the experiments with the hematocrit of the sealed blood as a parameter. The friction characteristics were also studied to find out the lubrication condition of the mechanical seal under blood sealing. In addition, the hemolytic properties of the sealed blood were examined to discuss the effects of those on the leakage characteristics.

\section{Nomenclature}

$\mathrm{Hb}_{b}$ hemoglobin concentration in blood [ppm]

$\mathrm{Hb} b_{p}$ hemoglobin concentration in plasma components [ppm]

MFI mechanical fragility index [\%]

$P_{b c}$ potassium ion concentration in blood cell components [ppm]

$P_{c} \quad$ potassium ion concentration in the C.W. [ppm]

$P_{p}$ potassium ion concentration in plasma components [ppm]

$S_{b c}$ sodium ion concentration in blood cell components [ppm]

$S_{c} \quad$ sodium ion concentration in the C.W. [ppm]

$S_{p} \quad$ sodium ion concentration in plasma components [ppm]

$V_{b} \quad$ volume of the sealed blood [ml]

$V_{b c} \quad$ volume of blood cell components [ml]

$V_{c} \quad$ volume of the C.W. [ml]

$V_{h b c}$ volume of the hemolyzed red blood cells [ml]

$V_{p} \quad$ volume of plasma components [ml]

$\Delta V_{b} \quad$ whole blood leakage [ml]

$\Delta V_{b c}$ blood cell component leakage [ml]

$\Delta V_{p}$ plasma component leakage [ml]

Subscripts

1 the start of the experiment

2 the time of the measurement of the blood leakage or the mechanical fragility index

\section{Materials and methods}

\subsection{Experimental apparatus}

Figures 1 and 2 show the schematic view of the experimental apparatus and the enlarged view of the sealing faces, respectively. The mechanical seal was composed of a rotating seal ring and a seat ring fixed to the housing. The seal ring and the seat ring were made of carbon graphite and silicon carbide, respectively. The torque meter was connected the shaft to the motor to

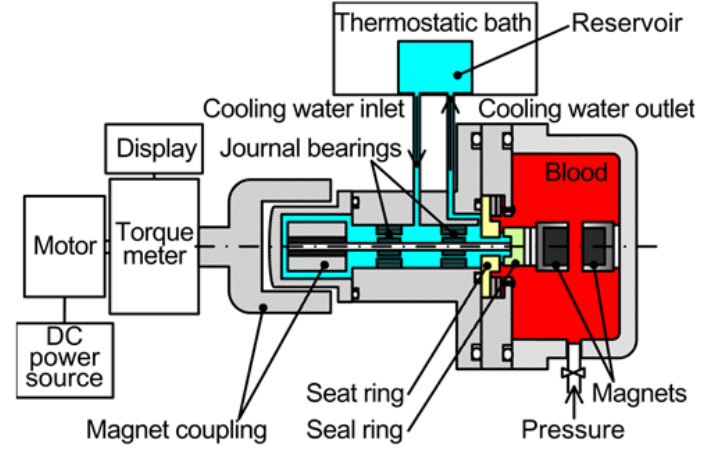

Fig. 1 Schematic view of the experimental apparatus

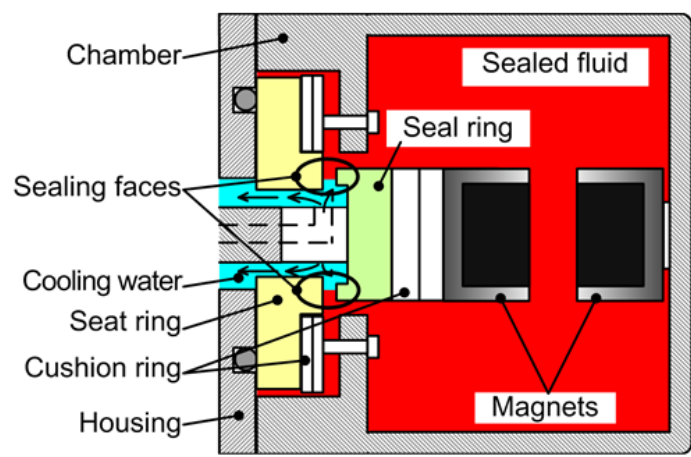

Fig. 2 Enlarged view of the seal ring and the seat ring

measure the friction torque in the sealing faces. Blood was sealed in the chamber. The contact force was given to the sealing faces with the repulsion between the magnets attached to the rotating shaft and in the chamber. Pure water was circulated at the inner side of the sealing faces as the C. W. and the reservoir of that was kept at the constant temperature.

\subsection{Hematocrit of the sealed blood}

Blood generally contains about $40-45 \%$ blood cell components and about 55-60\% plasma components [7]. Blood cell components mainly consist of red blood cells, and contain a few white blood cells and platelets. Therefore the hematocrit which shows the volume percentage of blood cell components in blood is regarded as the volume percentage of red blood cells. The hematocrit of the sealed blood was controlled in the experiments to prevent individual differences in the hematocrit.

3.3. Measurement of the blood cell component leakage and the plasma component leakage

A new measurement method of the blood cell component leakage and the plasma component leakage was recently proposed [6]. Each component leakage was obtained by measuring the increase in the potassium ion concentration and the sodium ion concentration in the $\mathrm{C}$. W. with an ion chromatograph. First, each ion concentration in each component was measured as shown in Fig. 3. When measuring each ion concentration, both components were diluted with pure water by 10,000 


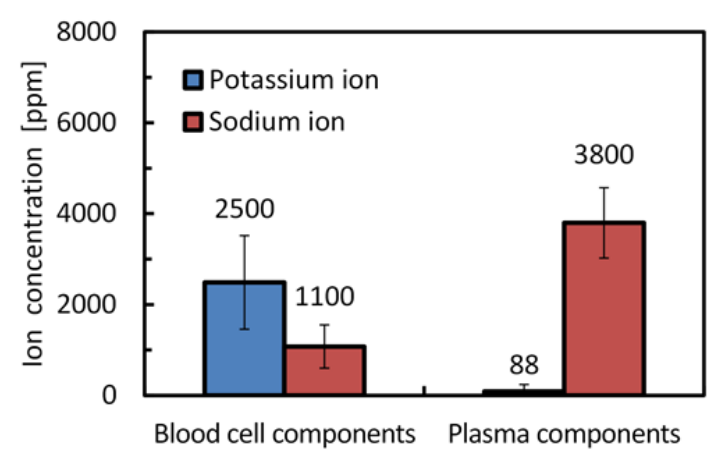

Fig. 3 Ion concentrations in blood cell components and plasma components

times to set the ion concentration in the measurable range of the ion chromatograph. Red blood cells were, therefore, hemolyzed due to the osmotic pressure difference between inner red blood cells and pure water. The values showed in Fig. 3 are 10,000 times as much as measurement results. Figure 3 presents that the potassium ion concentration in plasma components $P_{p 1}$ was small enough to be ignored compared to that in blood cell components $P_{b c 1}$. Consequently, assuming the sodium ion content and the potassium ion content in the experimental system at the time of the measurement does not change from those at the start of the experiment, Eq. (1) is given:

$$
\left\{\begin{array}{l}
P_{b c 1} V_{b c}+P_{c 1} V_{c}=P_{b c 2}\left(V_{b c}-\Delta V_{b c}\right)+P_{c 2}\left(V_{c}+\Delta V_{b c}\right) \\
S_{p 1} V_{p}+S_{b c 1} V_{b c}+S_{c 1} V_{c}=S_{p 2}\left(V_{p}-\Delta V_{p}\right) \\
+S_{b c 2}\left(V_{b c}-\Delta V_{b c}\right)+S_{c 2}\left(V_{c}+\Delta V_{p}+\Delta V_{b c}\right)
\end{array}\right.
$$

When measuring the potassium ion concentration and the sodium ion concentration in the C. W., red blood cells in the C. W. were hemolyzed since pure water was used as the C. W.. Equation (1) is, therefore, completed because each ion concentration in blood cell components, $P_{b c 1}$ and $S_{b c 1}$, and that in the C. W., $P_{c 1}, P_{c 2}, S_{c 1}$ and $S_{c 2}$, were measured under the same condition. In Eq. (1), assuming that $P_{b c 1}=P_{b c 2}, S_{p 1}=S_{p 2}$ and $S_{b c 1}=S_{b c 2}$, the blood cell component leakage $\Delta V_{b c}$ and the plasma component leakage $\Delta V_{p}$ are obtained as Eq. (2):

$$
\left\{\begin{array}{l}
\Delta V_{b c}=\left(\frac{P_{c 2}-P_{c 1}}{P_{b c 1}-P_{c 2}}\right) V_{c} \\
\Delta V_{p}=\left\{\left(\frac{S_{c 2}-S_{c 1}}{S_{p 1}-S_{c 2}}\right)-\left(\frac{S_{b c 1}-S_{c 2}}{S_{p 1}-S_{c 2}}\right)\left(\frac{P_{c 2}-P_{c 1}}{P_{b c 1}-P_{c 2}}\right)\right\} V_{c}
\end{array}\right.
$$

The whole blood leakage $\Delta V_{b}$ is given as Eq. (3):

$$
\Delta V_{b}=\Delta V_{b c}+\Delta V_{p}
$$

3.4. Measurement of the friction torque in the sealing faces

The friction torque in the sealing faces was measured to discuss the lubrication condition of the mechanical seal under blood sealing.

\subsection{Measurement of the mechanical fragility index}

Hemolysis is the phenomenon that red blood cells are damaged and that hemoglobin in red blood cells is discharged into surrounding fluid. Hemolysis is generated because of the passage of time, the osmotic pressure difference between inner and outer red blood cells. Physical loads also damage red blood cells and cause hemolysis. In this study, the mechanical fragility index MFI [\%] of the sealed blood after the measurement experiment of the blood leakage was measured as the hemolytic index to examine the effects of the hemolysis on the leakage characteristics of the mechanical seal. The mechanical fragility index MFI is defined as Eq. (4) which gives the ratio of the amount of hemolyzed red blood cells after the measurement experiments of the blood leakage to that of unhemolyzed red blood cells at the start of the experiment [8].

Table 1 Experimental conditions

\begin{tabular}{c|c}
\hline \hline Sealed fluid & Pig blood \\
\hline Hematocrit $[\%]$ & $0,10,20,30$ and 40 \\
\hline C. W. & Pure water \\
\hline Contact force $[\mathrm{N}]$ & 3.5 \\
\hline Contact pressure $[\mathrm{MPa}]$ & 0.083 \\
\hline Rotational speed $\left[\mathrm{min}^{-1}\right]$ & 2000 (Measurement of the blood leakage and the hemolytic index) \\
C. W. pressure $[\mathrm{kPa}]$ & $400-3000$ (Measurement of the friction torque) \\
\hline Chamber pressure $[\mathrm{kPa}]$ & 2.00 \\
\hline Flow rate of the C. W. $[\mathrm{ml} / \mathrm{min}]$ & 13.3 \\
\hline$R_{\mathrm{a}}$ of the seal ring $[\mu \mathrm{m}]$ & 4. \\
\hline$R_{\mathrm{a}}$ of the seat ring $[\mu \mathrm{m}]$ & 0.111 \\
\hline Temperature of the C. W. $[\mathrm{K}]$ & 0.028 \\
\hline Volume of the C. W. $[\mathrm{ml}]$ & 298 \\
\hline Volume of the chamber $[\mathrm{ml}]$ & 200 \\
\hline
\end{tabular}




$$
M F I=\frac{H b_{p 2}-H b_{p 1}}{H b_{b 1}-H b_{p 1}} \times 100
$$

\subsection{Experimental conditions}

Table 1 shows the experimental conditions. Pig blood was used as the sealed blood and the hematocrit was controlled to $40 \%$ corresponding to the hematocrit of human blood. The experiments were also conducted with the hematocrit of $0 \%$ to examine the effects of blood cell components and $10,20,30 \%$ to study each leakage characteristics of blood cell components and plasma components in detail. Blood was sealed at $13.3 \mathrm{kPa}$ corresponding to human blood. The leakages were measured at the speed of $2000 \mathrm{~min}^{-1}$. The friction torque was measured at the speeds of from $400 \mathrm{~min}^{-1}$ to 3000 $\mathrm{min}^{-1}$ in the steps of $200 \mathrm{~min}^{-1}$.

\section{Results and discussion}

4.1. Effects of the hematocrit of the sealed blood on the leakage characteristics

Figures 4 and 5 demonstrate the effects of the hematocrit of the sealed blood on each component leakage, $\Delta V_{b c}$ and $\Delta V_{p}$, and the whole blood leakage in 3 hours $\Delta V_{b}$, respectively. It was found out that the blood cell component leakage was not obtained when the hematocrit was set to $10 \%$. It was also revealed that the blood cell component leakage became larger with the increase of the hematocrit. On the other hand, the plasma component leakage and the whole blood leakage also increased as the hematocrit was increased from $10 \%$ to $40 \%$. As a result, the volume percentage of the blood cell component leakage in the whole blood leakage increased with the increase of the hematocrit as shown in Fig. 6. When changing the hematocrit from $20 \%$ to $30 \%$, the volume percentage of the blood cell component leakage in 3 hours remarkably increased by 7.7 times. In addition, the volume percentage of the blood cell component leakage increased with the passage of time with each of the sealed blood.

4.2. Effects of the hematocrit of the sealed blood on the friction characteristics

Figure 7 shows the effects of the hematocrit of the sealed blood on the friction torque. As the hematocrit was decreased, the friction torque became smaller. The friction torque also decreased with each of the sealed blood, as the rotational speed was increased. This is the typical trend under the mixed lubrication condition in the Stribeck curve. Assuming that the mechanical seal was operated under the mixed lubrication condition, the film thickness in the sealing faces was on the same order as the surface roughness, which was a submicron order. Also assuming that a red blood cell, having a width of 8 $\mu \mathrm{m}$ and a thickness of $2 \mu \mathrm{m}$, was larger than the film thickness, it is reasonable to say that hemolyzed red blood cells passed between the sealing faces as the blood cell component leakage. Since the viscosity of hemolyzed red blood cells is larger than that of plasma
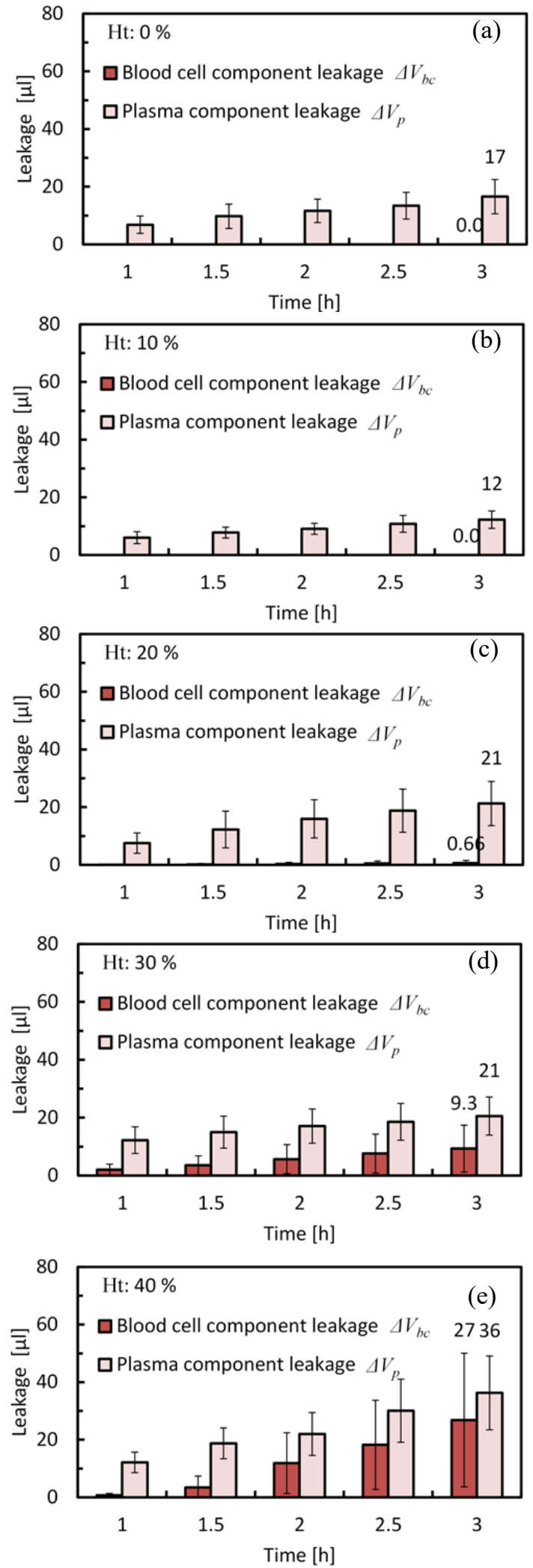

Fig. 4 Effects of the hematocrit of the sealed blood on the blood cell component leakage and the plasma component leakage

(a) Ht: $0 \%$, (b) $\mathrm{Ht}: 10 \%$, (c) Ht: $20 \%$, (d) Ht: $30 \%$, (e) Ht: $40 \%$ 


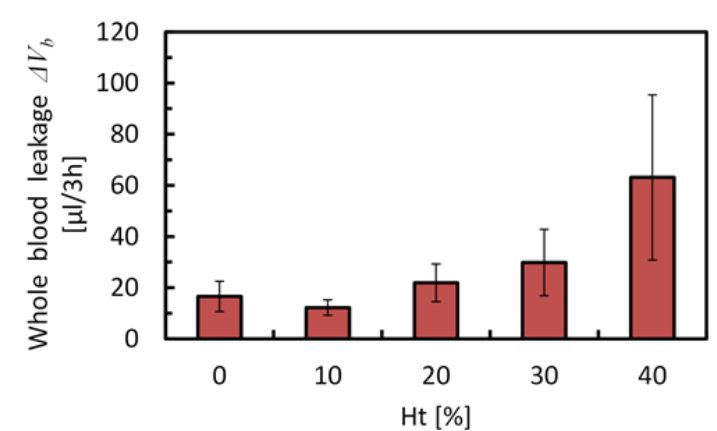

Fig. 5 Effects of the hematocrit of the sealed blood on the whole blood leakage in 3 hours

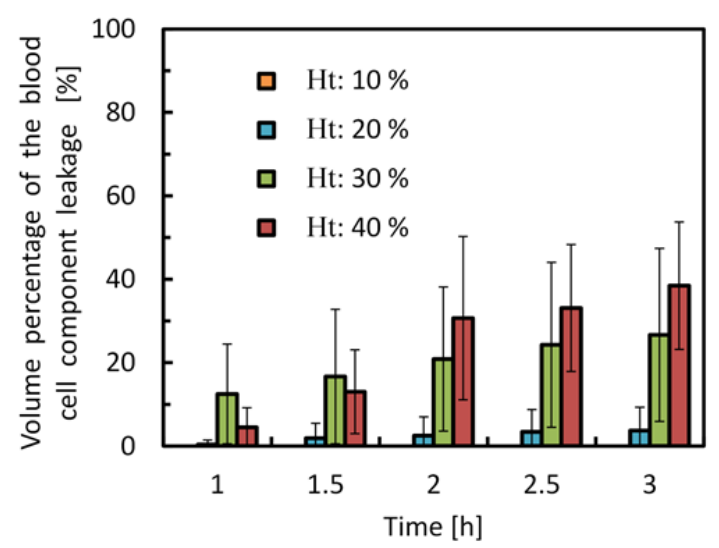

Fig. 6 Effects of the hematocrit of the sealed blood on the volume percentage of the blood cell component leakage

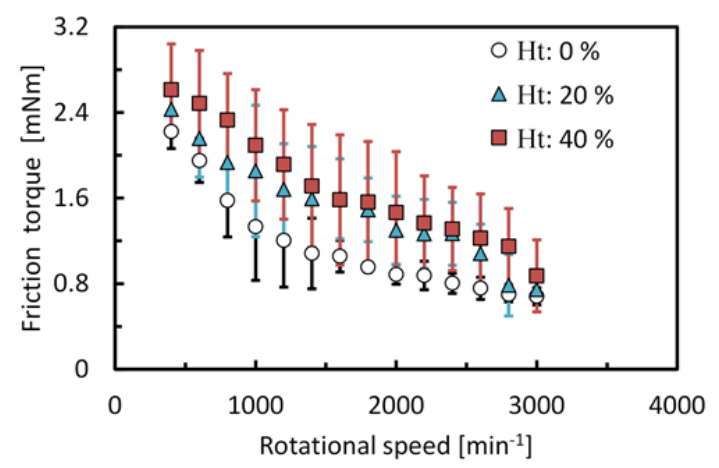

Fig. 7 Effects of the hematocrit of the sealed blood on the friction characteristics

components [9], it is considered that the friction torque becomes smaller with the decrease of hemolyzed red blood cells in the sealing faces. Therefore, since the volume percentage of the blood cell component leakage reduced with the decrease of the hematocrit as shown in Fig. 6, the friction torque is considered to decrease with the decrease of the hematocrit. As the hemolysis progress with the passage of time, it is considered that the volume percentage of the blood cell component leakage increased with the passage of time as shown in Fig. 6.

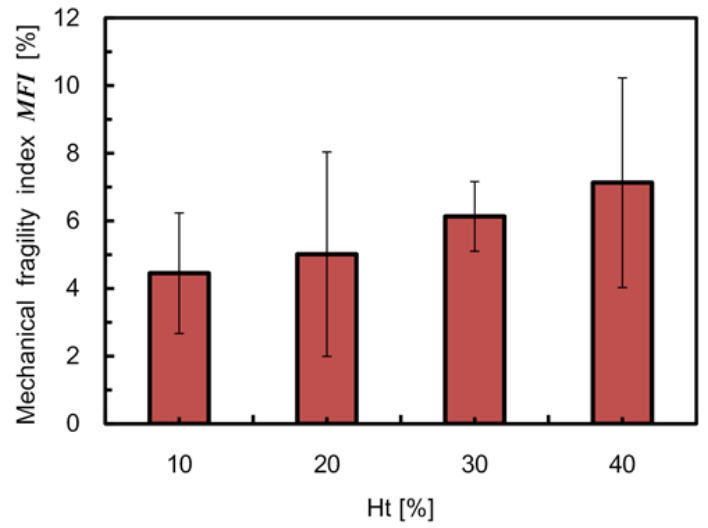

Fig. 8 Effects of the hematocrit on the hemolytic properties of the sealed blood

4.3. Effects of the hematocrit of the sealed blood on the hemolytic properties

Figure 8 demonstrates the mechanical fragility index MFI of the sealed blood in 3 hours. It was found that the mechanical fragility index increased with the increase of the hematocrit. Several reasons for the hemolysis are considered: the rotation of the shaft, the passage of time, the shear load in the sealing faces and the osmotic pressure difference between the $\mathrm{C}$. W. leakage and the inner red blood cells.

4.4. Effects of the hemolysis on the leakage characteristics

Figure 9 shows the relationship between the volume percentage of the hemolyzed red blood cells and the hematocrit of the sealed blood and Fig. 10 shows the relationship between the blood cell component leakage and the volume percentage of the hemolyzed red blood cells. Here, the volume of the hemolyzed red blood cells $V_{h b c}$ is obtained by Eq. (5):

$$
V_{h b c}=V_{b} \times \frac{M F I}{100} \times \frac{H t}{100}
$$

$H t$ means the hematocrit of the sealed blood [\%] and $V_{b}$ means the volume of the sealed blood. It was found out from Fig. 10 that the blood cell component leakage

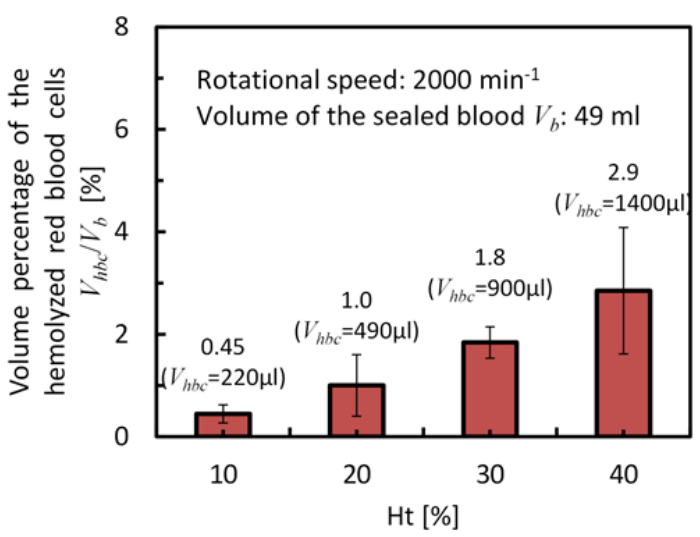

Fig. 9 Effects of the hematocrit on the volume percentage of the hemolyzed red blood cells 


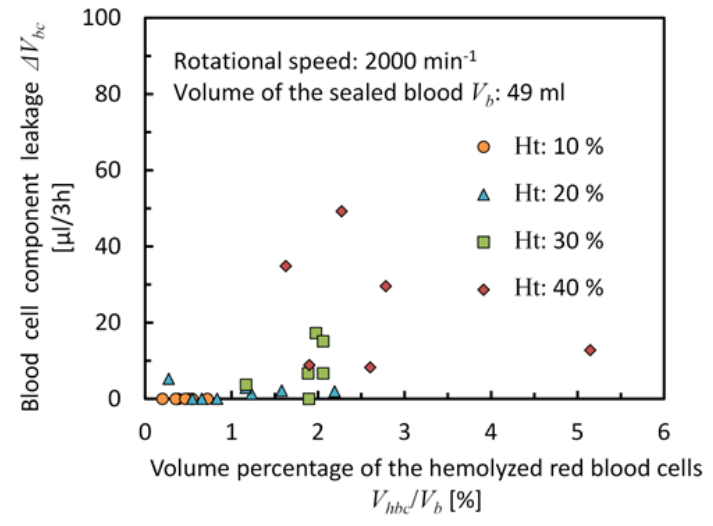

Fig. 10 Relationship between the blood cell component leakage and the volume percentage of the hemolyzed red blood cells

was detected when the volume percentage of the hemolyzed red blood cells was larger than about $1 \%$. It was also revealed from Fig. 9 that the volume percentage of the hemolyzed red blood cells increased with the increase of the hematocrit and that the volume percentage of the hemolyzed red blood cells was larger than about $1 \%$ when the hematocrit was $30 \%$ or $40 \%$ in most case. This, therefore, indicates that the blood cell component leakage with the hematocrit of $10 \%$ or $20 \%$ could not be detected in spite of the hemolysis generated in the sealed blood. It was also considered to be the reason why the blood cell component leakage increased with the increase of the hematocrit as shown in Fig. 4. From the above, the conditions when the blood cell component leakage was detected in this study were found out.

\section{Conclusions}

The effects of the hematocrit of the sealed blood on the leakage characteristics of blood cell components and plasma components were studied. The hematocrit was controlled to $0,10,20,30$ and $40 \%$. The friction characteristics of the mechanical seal and the hemolytic properties of the sealed blood were also examined. The conclusions are as follows:

1) The blood cell component leakage was not detected with the hematocrit of $10 \%$ and became larger with the increase of the hematocrit. The plasma component leakage and the whole blood leakage also increased as the hematocrit was increased from $10 \%$ to $40 \%$.

2) The volume percentage of the blood cell component leakage in the whole blood leakage increased with the passage of time with each of the sealed blood. It was also revealed that the volume percentage of the blood cell component leakage in 3 hours increased with the increase of the hematocrit. It remarkably increased by 7.7 times when the hematocrit was changed from $20 \%$ to $30 \%$.

3) As the rotational speed was increased, the friction torque became smaller with each of the sealed blood. This is the typical trend under the mixed lubrication condition in the Stribeck curve.

4) The mechanical fragility index of the sealed blood after 3 hours increased with the increase of the hematocrit.

5) The blood cell component leakage was detected when the volume percentage of the hemolyzed red blood cells in the sealed blood was larger than about $1 \%$ in this study. The volume percentage of the hemolyzed red blood cells was larger than about $1 \%$ when the hematocrit was $30 \%$ or $40 \%$ in most case.

\section{Acknowledgment}

This work was supported by JSPS KAKENHI Grant Number 26420087.

\section{References}

[1] Tomioka, J., Mori, T., Yamazaki, K., Akimoto, T. and Koyanagi, H., "In Vivo Study of the Performance of a Mechanical Seal Involving a Recirculating Cooling Water System Used in a Centrifugal Blood Pump," Japanese Journal of Artificial Organs, 29, 1, 2000, 47-50 (in Japanese).

[2] Yamazaki, K., Litwak, P., Tagusari, O., Mori, T., Kono, K., Kameneva, M., Tomioka, J., Umezu, M., Outa, E., Antaki, J. F., Kormos, R. L., Koyanagi, H. and Griffith, B. P., "An Implantable Centrifugal Blood Pump with a Recirculating Purge System (Cool-Seal System)," Artificial Organs, 22, 6, 1998, 466-474.

[3] Tomioka, J., Mori, T., Yamazaki, K. and Koyanagi, H., "Sealing Properties of Mechanical Seals for an Axial Flow Blood Pump," Artificial Organs, 23, 8, 1999, 708-711.

[4] Tomioka, J. and Miyanaga, N., "Effect of Surface Roughness of Mechanical Seals under Blood Sealing," Lubrication Science, 22, 2010, 443-452.

[5] Tomioka, J. and Oyabu, M., "Measurement of the Film Thickness in the Sealing Faces of the Mechanical Seal for a Blood Pump," 2011 Annual Conferences on Spring of Japan Society for Design Engineering, Tokyo, 2011, 37-38 (in Japanese).

[6] Tomioka, J., Oyabu, M., Fukui, K., Saito, K. and Miyanaga, N., "A Proposal for the Measurement Method of Plasma Component Leakage and Blood Cell Component Leakage in Mechanical Seals under Blood Sealing," Journal of Japan Society for Design Engineering, 50, 4, 2015, 194-199 (in Japanese).

[7] Guyton, A. C. and Hall, J. E. (edited by Mitarai, G.), "Textbook of Medical Physiology," Elsevier Japan Pub. Co. (2010), 437-438 (in Japanese).

[8] Kameneva, M. V., Garrett, K. O., Watach, M. J. and Borovetz, H. S., "Red Blood Cell Aging and Risk 
of Cardiovascular Diseases," Clinical Hemorheology and Microcirculation, 18, 1 (1998), 67-74.
[9] Kaibara, M. and Sakanishi, A. "Biorheology," Yoneda Pub. Co. (1999), 127-133 (in Japanese). 